Ann. Zootech., I972, 21 (I), 59-72.

\title{
ESSAI SUR LA CONCENTRATION DES ÉLÉMENTS NUTRITIFS CHEZ LA POULE PONDEUSE INTERACTION AVEC LE GÉNOTYPE ET INTÉRET DE L'ANALYSE INTRA-SOUCHE
}

\author{
J. GUILLAUME, J.-M. MELIN et J.-P. BOYER \\ avec la collaboration technique de Nicole Mithet, Michèle Sauvevr et Joëlle Bruère
}

Station de Recherches avicoles,

Centre de Recherches de Tours, I. N. R. A.,

37 - Nouzilly

RÉSUMÉ

Nous avons étudié les effets de la densité alimentaire $(2,30,2,54$ et 2,80 kcal énergie métabolisable/g) sur divers caractères ayant trait à la ponte de 3 populations différentes : deux souches expérimentales de type "ponte " et l'un de leurs croisements. Les pondeuses ( 1583 au total) ont été élevées en batteries de 20 à 54 semaines d'âge. La ponte globale n'est pas influencée de façon significative et on n'observe pas d'interaction génotype $\times$ densité alimentaire. Des effets de la densité alimentaire apparaissent cependant si on considère la consommation, la mortalité, le poids des pondeuses, la résistance à une vague de chaleur et la répartition des oufs au cours de la saison de ponte. Ces phénomènes s'expliquent en grande partie par une moins bonne régulation de l'ingéré énergétique en fonction de la densité alimentaire chez les animaux lourds.

Seule la souche la plus légère représentant plus de la moitié de l'effectif étudié justifie l'estimation de quelques paramètres génétiques. L'héritabilité de la ponte pour chaque période de contrôle apparaît très influencée par la densité alimentaire, l'aliment le plus dense donnant les plus fortes héritabilités en début de ponte et l'aliment le moins dense en fin d'expérience. La corrélation génétique entre le début et la fin de contrôle de ponte est également affectée, l'aliment le plus dense fournissant l'estimation la plus forte.

\section{INTRODUCTION}

Au fur et à mesure que les souches de volailles se différencient et que nos connaissances des besoins nutritifs s'améliorent, la nécessité d'adapter l'alimentation à la race, souche ou croisement, se pose avec une acuité croissante. 
On a d'abord recherché les répercussions du niveau de production, du poids de l'animal et de la température ambiante sur les besoins nutritionnels. BYERLY, I94I, a établi une équation permettant d'estimer les besoins énergétiques de la pondeuse en fonction du poids d'œufs pondus, du poids vif de l'animal et de son gain de poids. Combs, r968, a proposé des équations analogues pour les besoins de lysine et acides aminés soufrés.

En fait, les interactions entre génotype et taux protidique ou type de pondeuse et teneur en acides aminés sont souvent sans rapport avec les caractéristiques de production : Moreng et al., I964, DEATON et QUISENBERRY, I964, Lil.iIE et DENTON, I965, Balloun et Spekers, ig69. C'est seulement dans les expériences de Buys et Potgieter, i962, Harms, Damron et WALDroup, I966, que 1'on peut relier en partie un moindre besoin azoté à une ponte plus faible ou à une taille corporelle différente.

Un nombre plus réduit de travaux concerne les interactions type de pondeuse $\times$ taux énergétique (MCDANiEL et al., I957, BARTON et STEPHENSON, I960, LILLIE et DENTON, I965, Fox, I966). Ils démontrent l'existence de telles interactions mais n'en expliquent guère l'origine.

Dans une intéressante revue bibliographique, Morris, I969, étudie le rôle de la "densité alimentaire ": si l'on modifie parallèlement d'une part le niveau énergétique d'un aliment et d'autre part sa teneur en protéines, acides aminés indispensables, vitamines, etc., on agit sur la " densité alimentaire " sans toucher à l'équilibre des nutriments; les répercussions d'ordre économique en sont importantes : la production d'œufs est inchangée, la consommation exprimée en énergie n'est pas modifiée chez les pondeuses légères tandis qu'elle s'accroît avec la densité alimentaire chez les souches lourdes. Si l'on considère un ensemble de types de pondeuses d'origine et de potentiel génétique variés, il existe donc une interaction nette type de pondeuse $x$ densité alimentaire au niveau du " comportement alimentaire ». Cette interaction serait inexistante au niveau de la ponte : contrairement à une idée très répandue (DAwKINs, I964), les pondeuses légères à forte production n'auraient donc pas besoin d'aliments plus concentrés que les pondeuses plus lourdes.

Cependant, la conclusion de Morris, I969, provient essentiellement de la comparaison d'essais effectués sur des types différents et non d'essais où plusieurs types étaient éprouvés dans une même expérience. Il nous a donc semblé utile d'aborder à nouveau ce problème en choisissant 3 populations bien définies : deux souches et l'un de leurs croisements. D'un autre point de vue, il était intéressant d'étudier l'expression des différences génétiques intra-souche en fonction de l'aliment distribué. En effet, les paramètres utilisés au niveau de la sélection peuvent être modifiés selon les conditions zootechniques oì les données sont recueillies. Ainsi MÉrat, I96o, met en évidence l'influence de l'éclairement sur l'expression du génotype " précocité sexuelle ". Boyer, de LAAGE et CALET, I963, Mérat et Pron'homme, I g69, montrent l'effet du niveau énergétique de l'aliment sur l'héritabilité de divers critères de production du poulet de chair. Il apparaît ainsi opportun de tester les répercussions de la densité alimentaire sur quelques paramètres utilisés en sélection pour la ponte. 


\section{MATÉRIEL ET MÉTHODES}

Les deux souches pures sont deux souches expérimentales sélectionnées à la Station de Recherches avicoles de l'I. N. R. A. : d'une part la souche L 22 dérivant de la Leghorn blanche, d'autre part la M 55, souche synthétique plus lourde à œufs colorés. Leur croisement L $22 \times$ M 55 (L 25) manifeste un fort hétérosis en matière de ponte et de poids de l'œuf. Tous les animaux sont pédigree.

Les poulettes sont élevées au sol dans une poussinière obscure jusqu'à $\mathbf{r} 6$ semaines. Elles sont éclairées selon un programme " plat " ( 16 h par jour) et nourries ad libitum de la naissance à la fin de l'expérience et reçoivent les aliments expérimentaux à Ig semaines. La répartition dans les batteries de cages est faite de telle sorte qu'un nombre aussi voisin que possible de sujets dispose de chaque aliment à l'intérieur d'une même famille. Étant donné le nombre inégal et limité de poulettes il n'est cependant pas possible de répartir les représentants de chaque génotype en nombre rigoureusement égal dans chaque sous-groupe expérimental. Trois lots de poulettes descendant des mêmes parents sont utilisés successivement avec un décalage de 3 semaines. L'expérience porte donc sur 3 répétitions de 530 poulettes environ (tabl. I).

\section{TABLEAU I}

Effectifs et mortalité (p. roo) (chiffres en italique) dans les différents lots expérimentaux

\begin{tabular}{|c|c|c|c|c|c|c|c|c|c|}
\hline \multicolumn{2}{|c|}{ Régime } & \multirow{2}{*}{\multicolumn{2}{|c|}{ A }} & \multirow{2}{*}{\multicolumn{2}{|c|}{$\mathrm{T}$}} & \multirow{2}{*}{\multicolumn{2}{|c|}{$\mathrm{B}$}} & \multirow{2}{*}{\multicolumn{2}{|c|}{$\begin{array}{c}\text { Ensemble } \\
\text { des } 3 \text { aliments }\end{array}$}} \\
\hline Lot & Génotype & & & & & & & & \\
\hline 1 er $\operatorname{lot}$ & $\begin{array}{r}\text { L } 22 \\
\text { M } 55 \\
\text { L } 25\end{array}$ & $\begin{array}{r}105 \\
24 \\
34\end{array}$ & $\begin{array}{r}5,7 \\
12,5 \\
7,7\end{array}$ & $\begin{array}{r}107 \\
21 \\
38\end{array}$ & $\begin{array}{l}5,6 \\
4,2 \\
0,0\end{array}$ & $\begin{array}{r}107 \\
24 \\
38\end{array}$ & $\begin{array}{r}5,6 \\
20,8 \\
5,3\end{array}$ & $\begin{array}{r}319 \\
72 \\
115\end{array}$ & $\begin{array}{r}5,6 \\
12,5 \\
4,3\end{array}$ \\
\hline $2^{\mathrm{e}} \operatorname{lot}$ & $\begin{array}{r}\text { L } 22 \\
\text { M } 55 \\
\text { L } 25\end{array}$ & $\begin{array}{l}93 \\
31 \\
52\end{array}$ & $\begin{array}{l}5,4 \\
8,8 \\
5,8\end{array}$ & $\begin{array}{l}91 \\
31 \\
52\end{array}$ & $\begin{array}{l}6,4 \\
0,0 \\
9,6\end{array}$ & $\begin{array}{l}93 \\
35 \\
52\end{array}$ & $\begin{array}{r}10,8 \\
8,6 \\
7,7\end{array}$ & $\begin{array}{l}280 \\
103 \\
156\end{array}$ & $\begin{array}{l}7,5 \\
5,8 \\
7,7\end{array}$ \\
\hline $3^{e} 10 t$ & $\begin{array}{r}\text { L } 22 \\
\text { M } 55 \\
\text { L } 25\end{array}$ & $\begin{array}{l}97 \\
41 \\
42\end{array}$ & $\begin{array}{r}2,1 \\
12,2 \\
7,1\end{array}$ & $\begin{array}{l}96 \\
41 \\
49\end{array}$ & $\begin{array}{l}6,2 \\
4,9 \\
7,1\end{array}$ & $\begin{array}{l}97 \\
41 \\
41\end{array}$ & $\begin{array}{r}10,8 \\
14,2 \\
2,4\end{array}$ & $\begin{array}{l}290 \\
123 \\
125\end{array}$ & $\begin{array}{l}5,9 \\
9,8 \\
5,6\end{array}$ \\
\hline $\begin{array}{c}\text { Ensemble } \\
\text { des } \\
3 \text { lots }\end{array}$ & $\begin{array}{r}\text { L } 22 \\
\text { M } 55 \\
\text { L } 25\end{array}$ & $\begin{array}{r}295 \\
99 \\
133\end{array}$ & $\begin{array}{r}4,4 \\
11,1 \\
6,8\end{array}$ & $\begin{array}{r}297 \\
99 \\
132\end{array}$ & $\begin{array}{l}6,1 \\
3,0 \\
6,1\end{array}$ & $\begin{array}{l}297 \\
100 \\
131\end{array}$ & $\begin{array}{r}8,4 \\
13,0 \\
5,3\end{array}$ & $\begin{array}{l}889 \\
298 \\
396\end{array}$ & $\begin{array}{l}6,3 \\
9,1 \\
6,1\end{array}$ \\
\hline Total & & 527 & 6,3 & 528 & 5,5 & 528 & $8, j$ & 1583 & 6,8 \\
\hline
\end{tabular}

Les régimes alimentaires éprouvés comprennent le régime $\mathrm{T}$ (témoin) utilisé pour nourrir les poules des générations antérieures qui fournit 2,54 kcal d'énergie métabolisable par $g$ et I 4,4 p. roo de protéines brutes, et les régimes A et $\mathrm{B}$. Ces derniers ont une densité alimentaire inférieure et supérieure de ro p. Ioo respectivement (tabl. 2). Le nombre d'œufs pondus est enregistré dès le début de la ponte, journellement et individuellement pendant 23 semaines, puis regroupé par périodes correspondant sensiblement au $\mathrm{I} / 3$ de la durée de l'essai. Les oufs sont pesés à la $45^{\mathrm{e}}$ et $4^{6}$ semaine d'âge dans chaque lot. Un échantillon de poules est pesé en fin d'expérience. La consommation n'est enregistrée que globalement pour chaque aliment dans chaque lot.

Pour l'analyse statistique on considère que l'on a affaire à un dispositif factoriel $3^{4}=3$ génotypes $\times 3$ régimes $\times 3$ lots $\times 3$ périodes, le dernier facteur étant considéré comme hiérarchisé 


\section{TABLEAU I bis}

Degrés de liberté des composantes des variances et des covariances relatives aux différentes périodes

(Souche L 22)

\begin{tabular}{|c|c|c|c|c|c|c|c|c|c|c|c|c|}
\hline \multirow{3}{*}{$\begin{array}{c}\text { de } \begin{array}{c}\text { Source } \\
\text { variation }\end{array} \\
\text { Régime }\end{array}$} & \multicolumn{9}{|c|}{ Période de ponte } & \multirow{2}{*}{\multicolumn{3}{|c|}{ Poids de l'œuf }} \\
\hline & \multicolumn{3}{|c|}{$1^{\mathrm{re}}$} & \multicolumn{3}{|c|}{$2^{e}$} & \multicolumn{3}{|c|}{$3^{e}$} & & & \\
\hline & A & $\mathrm{T}$ & B & A & $\mathrm{T}$ & B & A & $\mathrm{T}$ & B & A & $\mathrm{T}$ & B \\
\hline $\begin{array}{l}\text { Intra-mères } \\
\text { Entre mères }\end{array}$ & 176 & 180 & 172 & 172 & 174 & $16^{\prime} \mathrm{k}$ & 169 & 169 & 159 & 126 & 124 & $11:$ \\
\hline Intra-pères ... & 71 & 67 & 70 & 71 & 67 & 69 & 70 & 67 & 69 & 65 & 62 & 65 \\
\hline Entre pères . & 37 & 37 & 39 & 37 & 37 & 39 & 37 & 37 & 39 & 37 & 39 & 39 \\
\hline Total. ....... & $28{ }^{\prime}$ & 284 & 281 & 280 & 278 & 272 & 276 & 273 & 267 & 228 & 223 & 218 \\
\hline
\end{tabular}

TABLEAU 2

Composition des régimes expérimentaux

\begin{tabular}{|c|c|c|c|}
\hline \multirow{2}{*}{ Composants } & \multicolumn{3}{|c|}{ Régimes } \\
\hline & A & $\mathrm{T}$ & $\mathrm{B}$ \\
\hline 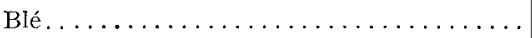 & 17,00 & 12,50 & - \\
\hline Avoine $\ldots \ldots \ldots \ldots \ldots \ldots \ldots \ldots$ & 45,50 & 7,00 & - \\
\hline Mais $\ldots \ldots \ldots \ldots \ldots \ldots \ldots \ldots \ldots$ & 5,65 & $4 \mathbf{4}, 75$ & 69,15 \\
\hline Son de blé $\ldots \ldots \ldots \ldots \ldots \ldots \ldots \ldots$ & 10,00 & 5,00 & - \\
\hline Farine de viaude $50 \% \ldots \ldots \ldots \ldots$ & 1,00 & 2,00 & 5,00 \\
\hline Tourteau de soja cuit $44 \% \ldots \ldots \ldots \ldots$ & 4,50 & 7,00 & 11,50 \\
\hline Tourteau de tournesol $42 \% \ldots \ldots \ldots$ & - & 5,00 & 5,00 \\
\hline Farine de luzerne déshydratée . . . . . . . . & 8,00 & 8,00 & 1,00 \\
\hline Phosphate bicalcique............... & 1,20 & 1,10 & 0,80 \\
\hline Carbonate de calcium .............. & 5,80 & 6,00 & 6,20 \\
\hline Bicarbonate de sodium ............ & 0,60 & 0,60 & 0,60 \\
\hline Complément minéral et vitaminique $* \ldots$ & 1,00 & 1,00 & 1,00 \\
\hline DL-méthionine $\ldots \ldots \ldots \ldots \ldots \ldots \ldots$ & - & - & 0,02 \\
\hline Énergie métabolisable $(\mathrm{kcal} / \mathrm{g}) \ldots \ldots \ldots \ldots$ & 2,29 & $2,5^{\prime}$ & 2,80 \\
\hline Protéines $(\mathrm{N} \times 6,25) \% \ldots \ldots \ldots$ & 12,95 & 14,43 & 15,94 \\
\hline dont $\int$ Lysine $(\%) \ldots \ldots \ldots \ldots \ldots \ldots$ & $0,5 / 4$ & 0,60 & 0,73 \\
\hline dont Méthionine + cystine $(\%) \ldots \ldots$ & 0,50 & 0,53 & 0,57 \\
\hline Calcium $(\%) \ldots \ldots \ldots \ldots \ldots \ldots \ldots$ & 2,48 & 2,70 & 2,83 \\
\hline
\end{tabular}

* Apportant pour $100 \mathrm{~kg}$ : Vitamine A : $800000 \mathrm{UI}$; Vitamine $\mathrm{D}^{3}$ : $100000 \mathrm{UI}$; Tocophérol : $3 \mathrm{~g}$; Acide nicotinique : $0,5 \mathrm{~g}$; BHT : $12,5 \mathrm{~g}$; Iodure de $\mathrm{K}$ stabilisé : $0,1 \mathrm{~g}$; Sulfate de manganèse : $35 \mathrm{~g}$; Hygromycine B:+. 
dans le $3^{\mathrm{e}}$ (Milliers, I967). Seuls sont retenus, parce qu'ils présentent un intérêt, les effets du génotype, du régime et des interactions génotype $\times$ régime, génotype $\times$ période, régime $\times$ période et génotype $\times$ régime $\times$ période. L'unité expérimentale choisie est la ponte d'un sous-ensemble correspondant à la combinaison génotype $\times$ régime $\times$ lot $\times$ période. En d'autres termes, l'analyse de variance est effectuée après approximation : le dispositif est considéré comme équilibré.

Enfin, l'estimation des paramètres héritabilité et corrélation génétique est établie par analyse hiérarchique de la variance et de la covariance, cas de sous-classes inégales (KING et HENDERSON, 1954). Le tableau I bis indique les degrés de liberté dans chaque cas. Mais, au préalable, pour corriger les différences entre lots, toutes les données brutes subissent une transformation de type probit, en choisissant pour paramètre de la loi normale de référence la moyenne ro et l'écarttype 3. L'unité d'échelle est donc le tiers d'écart-type ou tierçon.

\section{RÉSULTATS}

$\mathrm{I}^{\mathrm{O}}$ - L'ensemble des résultats concernant la ponte est consigné dans le tableau 3. Après analyse statistique, tableau 3 bis, il ressort que :

\section{TABLEAU 3}

Résultats obtenus sur la ponte: infuence du génotype et de l'alimentation

(Ensemble des 3 lots)

\begin{tabular}{|c|c|c|c|c|c|}
\hline \multirow{2}{*}{ Génotype } & \multicolumn{4}{|c|}{ Régime } & \multirow{2}{*}{ Moyenne } \\
\hline & & A & $\Gamma$ & B & \\
\hline \multirow{4}{*}{ L 22} & 1 & $2{ }_{4}, 5$ & 34,5 & 31,0 & 30,0 \\
\hline & 2 & 58,2 & 59,4 & 57,7 & 58,4 \\
\hline & 3 & 52,3 & 57,0 & 47,1 & 52,1 \\
\hline & $m$ & 45,0 & 50,3 & 45,3 & 46,8 \\
\hline \multirow{4}{*}{ M 55} & 1 & 38,3 & 40,1 & 40,7 & 39,7 \\
\hline & 2 & 62,9 & 62,6 & 61,4 & 62,3 \\
\hline & 3 & 54,9 & 52,5 & 50,0 & 52,5 \\
\hline & $m$ & 52,0 & 51,7 & 50,7 & 51,5 \\
\hline \multirow{4}{*}{ L 25} & 1 & 39,4 & 46,1 & 46,1 & 44,0 \\
\hline & 2 & 65,0 & 67,2 & 70,6 & 67,6 \\
\hline & 3 & 54,8 & 55,9 & 56,2 & 55,6 \\
\hline & $m$ & 53,1 & 56,4 & 57,8 & 55,7 \\
\hline \multirow{4}{*}{ Moyenne } & 1 & 34,1 & 40,2 & 39,4 & 37,9 \\
\hline & 2 & 62,1 & 63,1 & 63,2 & 62,8 \\
\hline & 3 & 54,0 & 52,5 & 51,1 & 52,5 \\
\hline & $m$ & 50,1 & 51,9 & 51,2 & 51,1 \\
\hline
\end{tabular}

Les données sont exprimées en pourcentage de ponte. Les chiffres 1, 2 et 3 figurant dans la $1^{\text {re }}$ colonne correspondent à la $1^{\mathrm{re}}$, la $2^{\mathrm{e}}$ et la $3^{\mathrm{e}}$ période de ponte $m$ indiquant la moyenne. 
a) les deux souches et leur croisement ont une production nettement distincte $(p<0, \mathrm{OI})$;

b) l'effet global de la densité alimentaire est nul ;

c) l'interaction génotype $\times$ densité alimentaire n'est pas significative : aucun régime ne favorise nettement une souche particulière.

\section{TABLEAU 3 bis}

Analyse de la variance des résultats obtenus sur la ponte

(Ensemble des 3 lots)

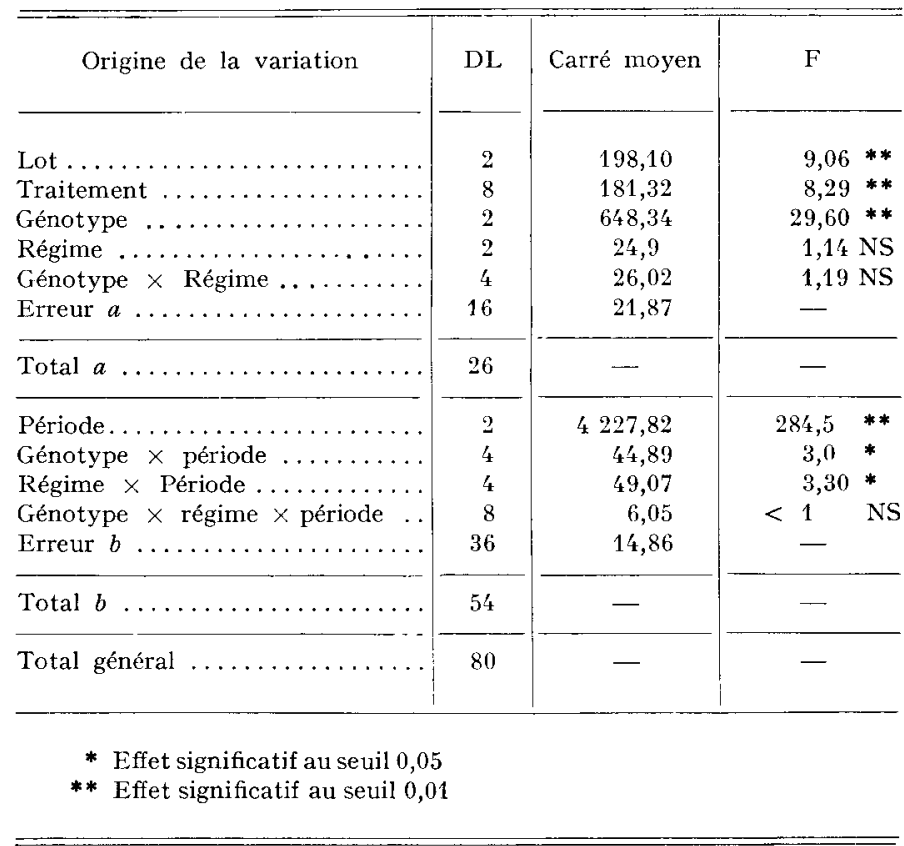

Si on décompose la production par période, on remarque, outre l'effet bien évident de la période de ponte :

d) une interaction génotype $\times$ période $(p<0,05)$ traduisant une entrée en ponte plus lente et une persistance meilleure dans la souche L 22 aux autres génotypes;

e) une interaction densité alimentaire $x$ période $(p<0,05)$. L'aliment à faible densité alimentaire donne de moins bons résultats durant la première période de ponte mais devient le meilleur durant la $3^{\mathrm{e}}$. I a densité alimentaire modifie donc la courbe de ponte (fig. I).

$2^{0}$ - En dehors de ces effets sur la production d'œufs, nous avons mis en évidence d'autres faits :

a) le poids de l'œuf, mesuré vers 45 semaines d'âge ne dépend que du génotype (tabl. 4); 


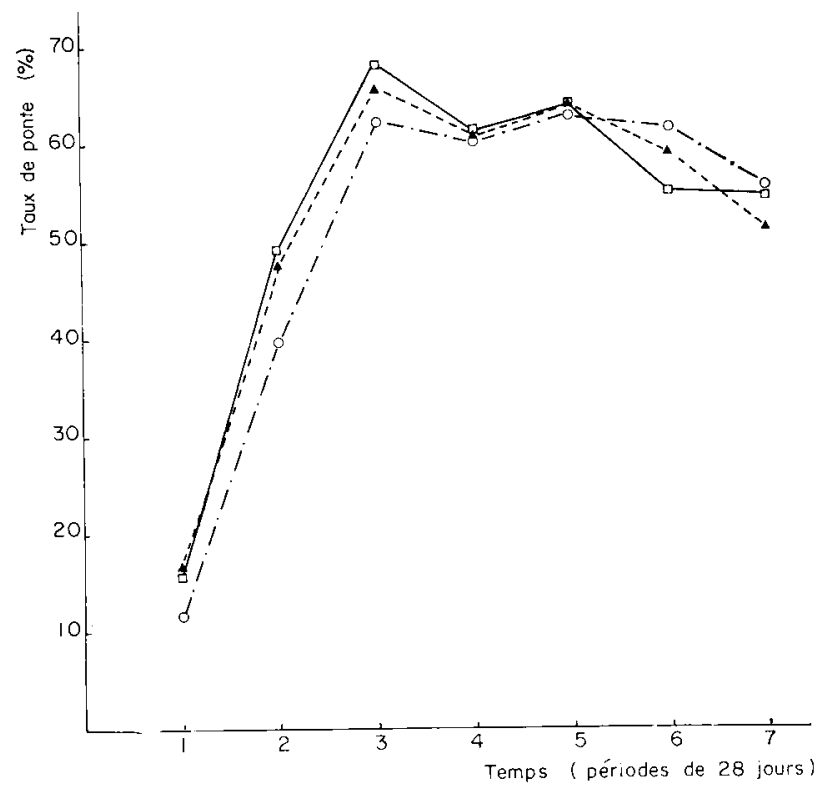

FIG. I. -- Influence de la densité alimentaive sur la forme de la courbe de ponte Exemple du Lot 1, tous génotypes confondus

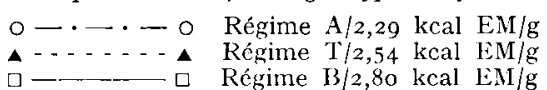

TABLEAU 4

Poids moyen de l'auf (g) à la $45^{\mathrm{e}}$ et la $46^{\mathrm{e}}$ semaine d'âge

(Ensemble des 3 lots)

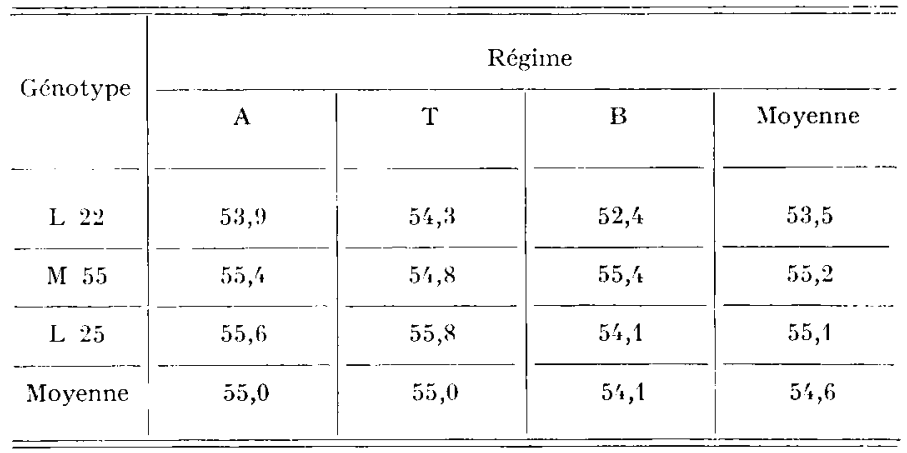

b) le poids des pondeuses à la fin de l'essai est fonction à la fois du génotype et du régime (tab1. 5). Il apparaît clairement que la densité alimentaire élevée alourdit les pondeuses et ce, de façon d'autant plus marquée que leur taille est plus grande;

c) la mortalité (tabl. I) est elle aussi influencée à la fois par le génotype, la souche M 55 étant plus sensible, et l'alimentation, les pertes les plus faibles correspondant à la densité alimentaire moyenne. Aucune interaction génotype $\times$ densité n'apparaît ; 


\section{TABLEAU 5}

Poids des animaux à 56 semaines (échantillon des 3 génotypes du lot I) et consommation journalière individuelle moyenne (lot $\mathrm{I}$, tous génotypes confondus)

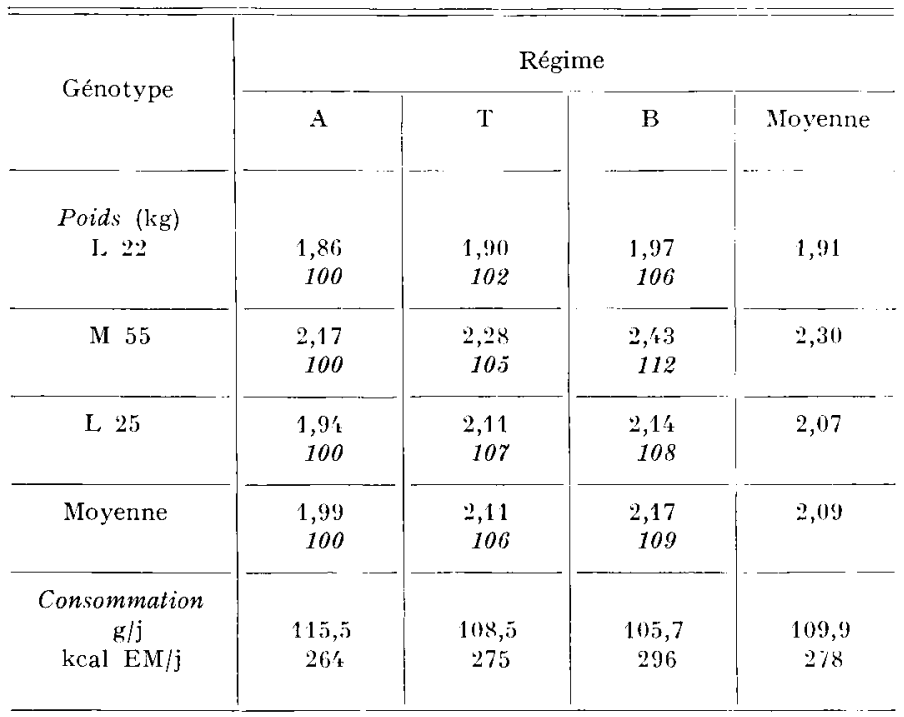

Les chiffres en italique indiquent pour chaque génotype le poids des poules des lots $\mathrm{B}$ et $\mathrm{T}$ en pourcentage de celui des poules du lot $\mathrm{A}$. Ils traduisent ainsi l'alourdissement des pondeuses dû à l'augmentation de la densité alimentaire.

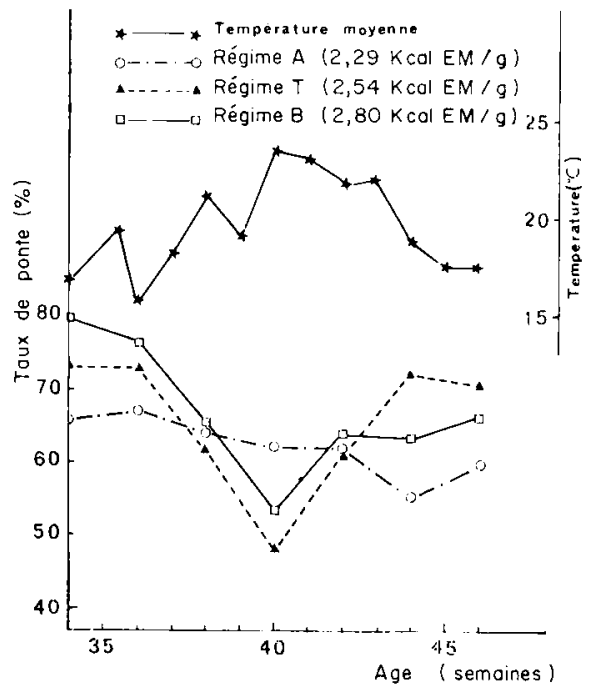

FIG. 2. - Influence de la densité alimentaire sur les effets du stress de chaleur. Exemple du Lot 1 croisement $L 25$ 


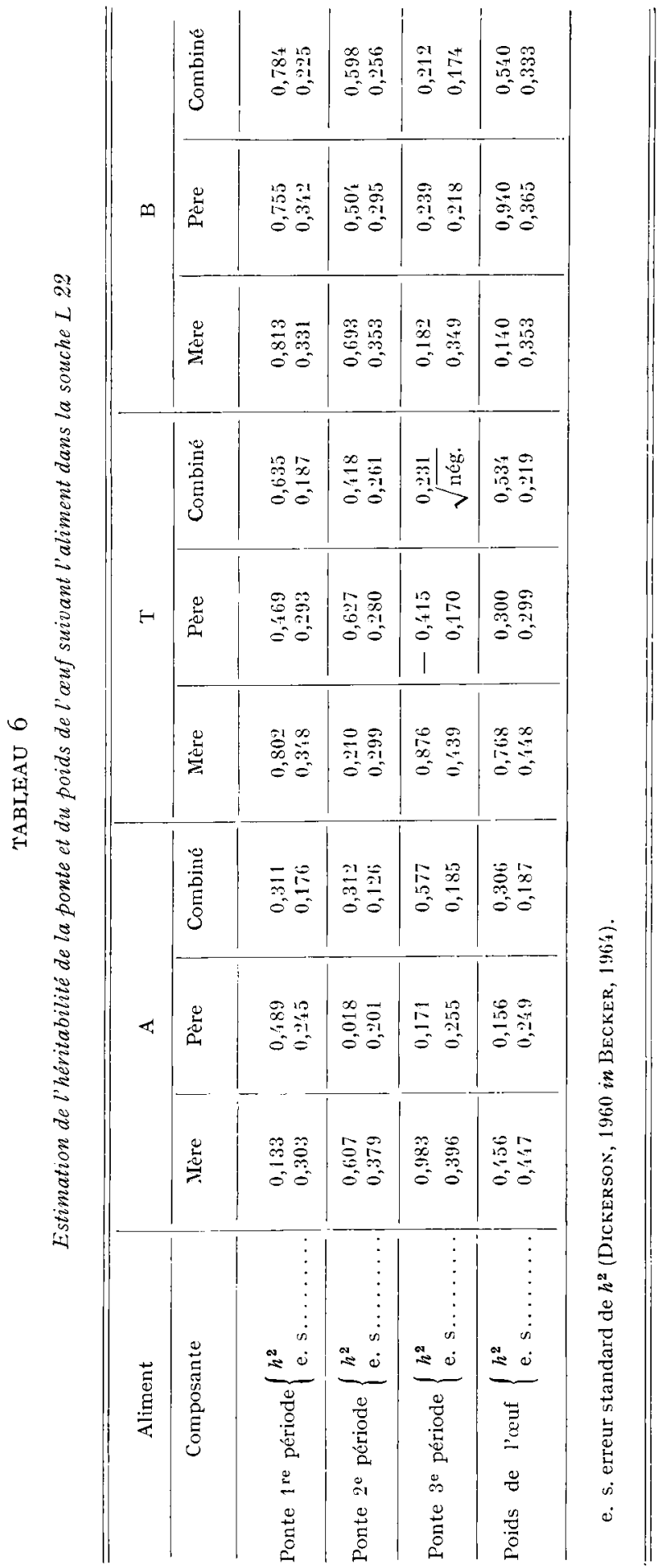


d) au cours d'une vague de chaleur (juillet-août I969), les pondeuses des régimes $B$ et ' $\mathrm{T}$ ont vu leur ponte diminuer tandis que celle du régime $A$ n'était pas affectée. Le "stress " dû à la chaleur ne s'est donc pas manifesté quand les poules étaient nourries avec un aliment très peu concentré (fig. 2).

$\left.3^{\circ}-a\right)$ Le tableau 6 indique les valeurs de l'héritabilité $\left(h^{2}\right)$ dans la souche L 22. On note une double tendance suivant la période de ponte et la densité alimentaire. L'estimation d'après la composante mère augmente avec le temps pour l'aliment $\mathrm{A}$ mais diminue pour l'aliment B ; elle augmente avec la densité alimentaire en $I^{\text {re }}$ période mais diminue en $3^{e}$ période. L'estimation d'après la composante père diminue en général avec le temps; elle augmente avec la densité alimentaire, d'autant plus nettement que la période considérée est plus précoce. Ce jeu antagoniste aboutit au total à une estimation composée de 1'héritabilité maximale en I $^{\text {re }}$ période pour $1^{\prime}$ aliment $B$, en $3^{\mathbf{e}}$ période pour l'aliment $\mathrm{A}$, le régime $\mathrm{T}$ étant intermédiaire mais plus proche de $B, 1 a 2^{e}$ période étant également intermédiaire mais plus proche de la $I^{r e}$.

On note, enfin, que les valeurs trouvées en régimes ' $T$ et $B$ pour les deux premières périodes et en régime $\mathrm{A}$ pour la $3^{\mathrm{e}}$ période sont considérablement plus élevées que les valeurs habituelles d'héritabilité citées pour les caractères de ponte $(20$ à 30 p. IOO contre 42 à 78 ici).

En ce qui concerne le poids de l'œuf, l'estimation d'après la composante père est multipliée par 6 quand on passe de l'aliment le moins dense à l'aliment le plus dense, tandis que la composante mère tend à varier en sens inverse.

b) Le tableau 7 indique les valeurs des corrélations phénotypiques et génotypiques entre la ponte au cours de la première période et les autres caractères contrôlés. D’une manière générale, les corrélations phénotypiques sont peu affectées par l'aliment ; il en va de même pour la corrélation génotypique entre la première et la deuxième période. Par contre, les autres corrélations génotypiques entre $\mathrm{I}^{\mathrm{re}}$ et $3^{\mathbf{e}}$ périodes comme entre ${ }^{r e}$ période et poids de l'œuf sont sensiblement diminuées en présence de 1'aliment $\mathrm{A}$.

\section{DISCUSSION}

Un certain nombre de résultats obtenus dans cet essai traduisent simplement des différences génétiques. L'effet "génotype " reflète le potentiel génétique des souches ainsi que 1'hétérosis. L'interaction génotype $\times$ période provient également de la sélection subie par les souches parentales : la souche $\mathrm{L} 22$ est sélectionnée pour une ponte élevée en début de production, la souche M 55 pour une meilleure persistance.

L'absence d'interaction génotype $x$ densité alimentaire dans le domaine de la ponte et du poids de l'œuf est en accord avec la conclusion de MorRIs, Ig69. Tant que la densité alimentaire ne dépasse pas les limites extrêmes de 2,33 et 3,40 kcal EiM/g, elle n'affecte ni la ponte ni (généralement) le poids de l'œuf, quelle que soit la souche testée.

L'existence d'une interaction génotype $\times$ densité sur le poids vif des pondeuses est également en accord avec cette conclusion : la souche la plus lourde (M 55) ajuste vraisemblablement plus mal sa consommation à la densité alimentaire, ingérant davantage de calories avec les régimes les plus denses. Remarquons, d'ailleurs, que ce 


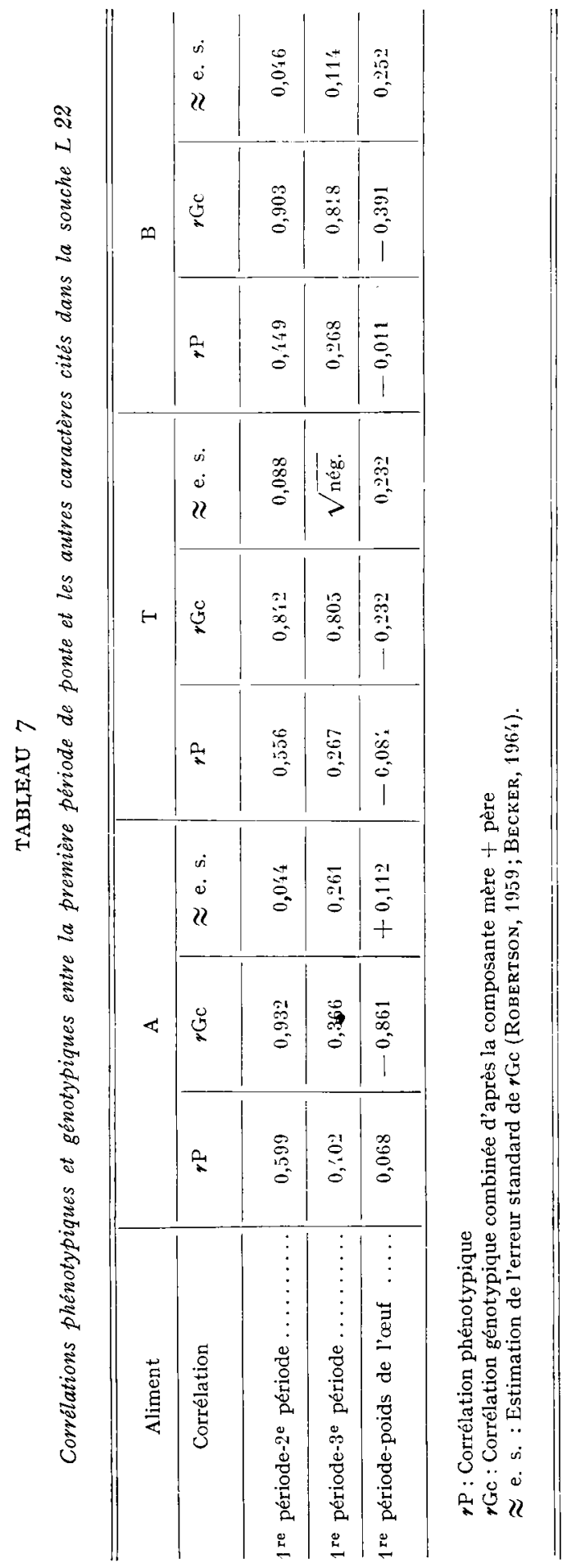


phénomène ne résulte sans doute pas de la densité alimentaire en soi mais du taux énergétique (DONALdoson et GoRdon, Ig60).

Plus originaux sont les effets de la densité alimentaire sur la forme de la courbe de ponte et sur les "stress " de chaleur. Certes, la réponse aux "stress " peut dépendre du niveau de production. Néanmoins, les deux effets précédents peuvent s'expliquer par le relai de la consommation. Les animaux recevant l'aliment à $2,30 \mathrm{kcal} / \mathrm{g}$ (considéré comme taux marginal) n'arrivent pas à couvrir leut besoin de production d'œufs et de croissance lors de l'entrée en ponte; en fin de ponte, ils bénéficient, au contraire, d'un moindre engraissement et, peut-être, d'une moindre "fatigue physiologique ". Les mêmes animaux, moins gras, résisteraient mieux à la chaleur. L'effet bénéfique des taux énergétiques élevés par temps froid a été souvent signalé (HIL,L I956, KURNICK et al., I96I) alors que l'effet bénéfique des taux énergétiques bas que nous observons ici ne l'a jamais été à notre connaissance. Les deux phénomènes, qui sont sans doute de même nature, paraissent plus marqués avec les souches lourdes.

$\mathrm{Si}$ on se borne aux performances enregistrées, les interactions génotype $x$ densité alimentaire apparaissent donc comme faibles malgré la diversité des potentiels génétiques et de l'origine des pondeuses. Il ne semble pas certain que les aliments peu denses favorisent les animaux lourds. Néanmoins, ces régimes peuvent assurer en plus d'une limitation de l'embonpoint, une régularisation de la courbe de ponte. Ce dernier phénomène mériterait confirmation sur des animaux plus productifs. L'interaction période de ponte $x$ densité alimentaire laisse penser qu'il serait peutêtre judicieux d'ajuster le niveau énergétique à la saison de ponte comme on a adapté le niveau azoté. Ce nouveau type d'alimentation en phase a déjà été éprouvé chez des pondeuses de type Leghorn où il est décevant (Summers, Pepper et Moran, r969). Il pourrait, cependant, se révéler avantageux chez des animaux plus lourds surtout dans le cas où une température élevée provoquerait des chocs plus faibles avec les densités alimentaires faibles.

L'intérêt de l'utilisation de régimes à densité variable est bien plus manifeste quand on considère les paramètres génétiques. Enn ce qui concerne la variabilité intra-souche, il est d'abord intéressant de noter qu'en milieu alimentaire $B$, il est plus avantageux d'améliorer la ponte en $3^{\mathrm{e}}$ période par sélection indirecte sur la I $^{\text {re }}$ période que par sélection directe, à différentiel de sélection égal.

On a en effet dans ce cas :

$$
r_{\mathrm{G}} h_{1}=0,82 \sqrt{0,784}=0,726 \quad h_{3}=\sqrt{0,2 \mathbf{I} 2}=0,460
$$

Par contre, en milieu alimentaire $A$, la diminution de la corrélation génotypique entre la $I^{\text {re }}$ et la $3^{\mathrm{e}}$ période donne l'avantage à la sélection directe sur les deux périodes de ponte. On a en effet dans ce cas :

$$
r_{\mathrm{G}} h_{3}=0,37 \sqrt{0,577}=0,28 \mathrm{r} \quad h_{1}=\sqrt{0,3 \mathrm{Ir}}=0,558
$$

Évidemment, il n'est pas possible de généraliser les résultats obtenus sur les échantillons de la présente expérience, mais on peut les considérer comme indicatifs d'éventualités qui peuvent se présenter en sélection lorsqu'on modifie les conditions d'élevage des animaux. En effet, cet essai montre que malgré l'absence d'interaction globale génotype $x$ aliment, l'analyse intra-souche révèle l'incidence de la densité alimentaire sur l'expression des différences génétiques régissant la variabilité des caractères à améliorer. 
De telles conditions d'élevage sont susceptibles de modifier la problématique de la sélection, justifiant, par exemple, la distribution de la souche en plusieurs populations soumises à des milieux de sélection spécialisés (milieu B pour la ponte précoce, milieu A pour la ponte tardive) ou encore, le rejet d'une sélection directe au profit d'une sélection indirecte (cas de la ponte tardive en aliment B).

La tactique du sélectionneur ne peut donc pas être systématique et doit s'inspirer de contingences révélées par l'analyse de chaque cas.

Les valeurs très élevées de $h^{2}$ obtenues ici indiquent que le gain génétique peut être accru d'une manière élégante par le jeu d'un milieu qui révèle des différences génotypiques cachées, sans recourir à l'intensité de sélection qui suit une loi de rendement décroissant.

$$
\text { Rę̧u pour publication en août } 1971 .
$$

\section{SUMMARY}

INTERACTION BETWEEN THE NUTRIEN'T DENSITY

OF THE DIETS AND THE GENOTYPE OF THE LAYING HEN. ADVANTAGE OF INTRA-STRAIN ANALYSIS

The present study deals with the effect of the nutrient density $(2.30-2.54$ and 2.80 kcal metabolizable energy $/ g$ ) on various characters related to the laying performances of 3 different populations : two experimental strains of the "laying type " and one of their crosses. The laying hens ( 1583 birds) were kept in batteries between 20 and 54 weeks of age. The laying performances were not significantly affected and no interactions between the genotype and the nutrient density was noticed. However ; food intake, mortality, weight of the laying hens, resistance to a period of hot weather and the distribution of the eggs during the laying season appeared to be influenced by the nutrient density. These phenomena may, to a great extent, be explained by a bad regulation of the energy intake according to the nutrient density.

Only the lightest strain, constituting more than half of the animals studied, was used to estimate some genetic parameters. The heritability of the laying performances for each testing period seemed to be greatly affected by the nutrient density. The highest heritabilities were attained with the largest dictary level at the beginning of the laying period and with the lowest dietary level at the end of this period. The genetic correlation between the performances at the beginning and the end of the test period was also affected, the highest dietary density resulting in the highest estimation values.

\section{RÉFÉRENCES BIBLIOGRAPHIQUES}

13alioun S. L., Speers G. N., r969. Protein requirements of laying hens as affected by strain. Poult. Sci., 48, II75-II 88 .

Barton L., Stephenson E. L., I960. Dietary interrelationships betwen levels of protein, fat and supplementary methionine hydroxyanalogue for both egg type and meat type hens Poult. Sci., 39, 1233. (Abstr.).

BECKER W.A., I964. Manual of procedures in quantitative genetics, 7o pp + VIII. Mimeograph. Washington St. Univ. Pullman. Washington.

Boyer J.-P., LaAge X. de, Calet C., 1963. Influence of feed on the heritability of some chicken traits. XIth Int. Conf. Genet., 1, 262 (Abstr.).

Buys G.S., Porgieter D. J. J., I962. A nitrogen balance study of the protein level required in laying rations. Dpt. Agric. Tech. Serv. Pretoria. Techn. comm., 8, 32 p.

Byerly T. C., I94I. Feed and other costs of producing market eggs. Md. Agr. Exp. Sta. Bull., 346. 
Comss G. F., 1968. Amino acid requirements of broilers and laying hens. Proc. U. Md. Nutr. Conf., 86-96.

DAwkins C. W. C., I964. A review of recent investigation on high and low energy rations for the laying hen. Naas, Quart. Rev., 65, 6-I3.

Deaton J. W., Quisenberry J. M., I964. Effect of dietary protein levels on performance of four commercial strains of egg-type birds Poult. Sci., 43, I 3 I2. (Abstr.).

Fox J., 1966. Sources of energy for laying birds rations (Abstr.). World's Poult. Sci. J., 22, I57.

Harms R. M., Damron B. L., Waldroup P. W., I966. Influence of strain or breed upon the protein requirement of laying hens. Poult. Sci., 45, 272-275.

Hill F. W., Anderson D. L., Dansky L. M., 1956. Studies of Energy requirements of chickens. 3. The effect of dietary energy level on the rate and gross efflciency of egg production. Poult. Sci., 35, 54-59.

KING S. C., Henderson C. R., 1954. Variance components analysis in heritability studies. Poult. Sci., 33, 147-154.

Kurnick A. A., Minds M. 13., Pasvogei, M. W., Reid B. L., ig6I. Dietary energy levels for laying hens as related to age and environmental temperatures. I. - Effect on egg production, body weight and feed conversion. Poult. Sci, 40, 1483-149I.

Lillie R. J., Denton C. A., I965. Protein and energy interrelationships for laying hens. Poult. Sci., 44, 753-76r.

McDaniel A. H., Price J. D., Quisenberry J. H., Reid B. L., Couch J. R., ig57. Effect of energy and protein level on cage layers. Poult. Sci., 36, 850-854.

MÉrat P., ig6o. L'héritabilité de la ponte d'hiver des poulettes recevant ou non un éclairement supplémentaire. Ann. Zootech., 9, 24I-254.

Mérat P., Pron'homme J., I969. Héritabilités et interactions génotype $\times$ régime pour le poids à 8 semaines de poulets en présence de deux aliments. Ann. Génét. Sél. anim., 1, 299-3or.

Milniers C., r967. Les modèles mathématiques dans l'analyse de variance. Brochure Séminaire de biométrie. Nancy, 1967, $30 \mathrm{p}$.

Moreng R. E., linos H. L., Whittet W. A., Miller B. F., rg64. An analysis of strain response to clietary protein levels. Poult. Sci., 43, 630-638.

Morris T. R., ig69. Nutrient density and the laying hen. Poc. 3rd Nutr. Conf. for feed manufacturers, $L$. of Nottingham, I03-II4.

RoBertson A., 1959. The sampling variance of the genetic correlation coefficient. Biometrics, 15, 469-485.

Summers J. D., l'eppir W. F., Moran li. T., r969. Use of amino acid unbalanced and low protein starting rations for the rearing of egg production type pullets and subsequent performance of these pullets when placed on laying rations of varying protein levels. Poult. Sci., 48, r35 I-I 358 . 